\title{
Urinary tract infections in Multiple Sclerosis
}

Véronique Phé, ${ }^{1,2 *}$ Mahreen Pakzad, ${ }^{1}$ Carmel Curtis,${ }^{3}$ Bernadette Porter, ${ }^{4}$ Collette Haslam, ${ }^{1}$ Jeremy Chataway, ${ }^{4}$ Jalesh N. Panicker ${ }^{1}$

${ }^{1}$ Department of Uro-Neurology, The National Hospital for Neurology and Neurosurgery and UCL Institute of Neurology, Queen Square, London, UK

${ }^{2}$ Pitié-Salpêtrière Academic Hospital, Department of Urology, Assistance Publique-Hôpitaux de Paris, Pierre and Marie Curie Medical School, Paris 6 University, Paris, France

${ }^{3}$ Department of Clinical Microbiology, University College London Hospital, London, UK

${ }^{4}$ Queen Square Multiple Sclerosis Centre, The National Hospital for Neurology and Neurosurgery and UCL Institute of Neurology Queen Square, London, UK

* Corresponding author:

Véronique Phé, MD

Department of Uro-Neurology

The National Hospital for Neurology and Neurosurgery

Queen Square

London WC1N 3BG

United Kingdom

Email: phe.veronique@gmail.com

Phone: +33663231534

Keywords: multiple sclerosis; urinary tract infection; recurrent; urinary dipstick, culture; relapse 


\section{Abstract}

Background: Urinary tract infections (UTIs) are commonly reported by people with multiple sclerosis (PwMS) and have a significant impact on quality of life.

Objective: To provide an overview of the problem of UTIs in PwMS and offer a practical approach for the diagnosis and management.

Methods: A review of the literature through a Pubmed search up to October 2015 was performed using the following keywords: multiple sclerosis, neurogenic bladder, urinary tract infections, relapse, dipsticks, culture, recurrent, prevention.

Results: Consensus now define symptomatic UTI by a positive urine culture defined by $>10^{5}$ colony forming units $(\mathrm{CFU}) / \mathrm{mL}$ or $>10^{4} \mathrm{CFU} / \mathrm{mL}$ if a urethral catheter urine sample is taken, or any count of bacteria in a suprapubic bladder puncture specimen, both in addition to symptoms including fever, pain, changes in lower urinary tract symptoms or neurological status. Urinalysis is useful to exclude a UTI (negative predictive value 95\%) however on its own is insufficient to confirm a UTI, for which urine culture is required. Experts advise Asymptomatic UTIs should not be treated except in the context of an acute relapse. Currently, from international guidelines there is no validated stategy to prevent recurrent UTIs in PwMS. Conclusion: This review provides an overview of the diagnosis, treatment and prevention of UTIs in the setting of MS.

Word cound: abstract (200), text (2953) 


\section{Introduction}

Urinary tract infections (UTIs) commonly occur in multiple sclerosis (MS). Though a figure of $30 \%$ is often cited, estimates vary between 13 to $80 \%$ per patient life and this may be put down to the different patient cohorts studied [1]. However this is also likely to reflect the lack of a consensus definition or diagnostic criteria for a UTI in patients with neurological disorders such as MS [1]. In general, the likelihood of infection is greater with longer disease duration and higher EDSS score [2],[3], though UTIs may occur at any time point during the course of the disease [1]. Urinary tract infections may exacerbate MS related lower urinary tract (LUT) symptoms such as urinary urgency, frequency and incontinence.

Urinary tract infections are ranked amongst the top three reasons for hospitalization, being responsible for $30-50 \%$ of all in-patient admissions of PwMS [4],[5]. A recent audit across a London region estimated annual costs above $£ 800,000$ and was driven by a relatively small number of PwMS with frequent admissions. A prospective study of 73 patients with relapseremitting MS demonstrated an increased risk of exacerbation around the time of infections, particularly UTI [6] and that infection-related relapses were associated with more sustained neurological deficits and worse quality of life compared to exacerbations not associated with infections, due to the burden of frequent hospitalizations and worsened LUT symptoms [6],[7]. There is also evidence to suggest that UTIs were a significant predictive factor for death in MS [3] and a retrospective analysis of a large validated UK primary care database [3] found that survival in MS was influenced not only by the underlying disease, but also by other comorbidities such as UTIs, as well as other infections, heart disease and cancer, and lifestyle factors such as smoking and alcohol abuse.

Despite the burden of this problem, there is relatively little guidance around the evaluation and management of UTIs in PwMS [1],[7],[8]. So we provided an overview of the problem of UTI in MS by reviewing the existing evidence on the subject. Based on this review of the 
literature and on our practice in a centre of excellence, we offered a practical approach for the diagnosis, treatment and prevention of UTIs in PwMS.

\section{Diagnosis of UTI}

\section{Symptoms of UTI}

Recognising UTI in PwMS can be difficult since PwMS suffering from UTI may not report the symptoms typically associated such as fever, rigor, suprapubic or flank pain, urinary urgency, frequency or worsening urinary incontinence and haematuria.. Conversely, symptoms that my trigger suspicion of UTI in the general population, such as urinary urgency, frequency and incontinence may reflect chronic LUT dysfunction in PwMS and not signify infection [9].

A UTI may need to be suspected in the presence of vague non-specific symptoms such as back or abdominal discomfort, reduced appetite or lethargy, deterioration of neurological status, leakage between intermittent self-catheterisation (ISC), catheter blockage or the report of a cloudy urine with increased odour [10]. These symptoms are highly sensitive (77-95\%) for a UTI, however have low specificity $(<50 \%)[11]$.

\section{Urinalysis}

Urinalysis (urine dipstick test), provides a rapid method of screening for UTIs. The test for leukocyte esterase detects the presence of azurophilic granules of monocytes and granulocytes (neutrophilic, eosinophilic and basophilic) whereas the test for nitrites is a rapid screening method for possible infections caused by nitrate-reducing bacteria such as Escherichia coli, Enterobacter, Klebsiella, Citrobacter and Proteus. 
The presence of nitrites in the urine has a sensitivity of $45-60 \%$ and specificity of $85-98 \%$ for a UTI, leukocyte esterase test positivity a sensitivity of $48-86 \%$ and specificity of $17-93 \%$, and combined nitrites and leukocyte esterase test positivity (with one or both showing a positive result) a sensitivity of $75-92 \%$ and specificity of $65-87 \%$ depending on the study population and independently of the method of urine collection, as reported by a metaanalysis which included general population, children, pregnant women, elderly patients, urological patients and surgical patients and patients with spinal diseases [12]. However specific data regarding to MS population are lacking.

There are limitations to the use of urine dipsticks. Leukocyte esterase activity is not specific for a UTI and may be positive (and nitrites negative), in patients using a urinary catheter in the absence of UTI, or in patients already using antibiotics. Other causes of iselated leukocyturia (define here?) include balanitis, urethritis, tubereulosis, bladder tumours, viral infections, nephrolithiasis, foreign bodies, exercise, glomerulonephritis, and corticosteroid and cyclophesphamide use. On the other hand, not all bacteria reduce nitrates in the urine to nitrites, and relying on urinalysis alone may miss infections due to Enterococcus.

In a meta-analysis of 70 studies evaluating the diagnostic accuracy of urinalysis, Devillé et al. concluded that testing with urinalysis was useful to exclude UTI in all groups including neurological patients, if the results for nitrites or leukocyte esterase testing were negative [12]. Indeed, a negative predictive value of $95 \%$, was reported when the combined nitrite and leukocyte esterase test was measured against culture [13].

Therefore, urinalysis for the presence of nitrites and leukocyte esterase is useful to rule out an infection (negative predictive value 95\%). However, since the negative predictive value is not as $100 \%$, it is advisable to send a urine culture if there is a high clinical suspicion for a UTI such as malodourous or turbid urine, despite a negative dipstick. 
Urinalysis has a relatively low ability to diagnose an infection (positive predictive value $<76 \%$ ), thus, if urinalysis is positive, this should be followed up with urine culture to confirm whether indeed an infection is present. Figure 1 gives a suggested algorithm.

\section{Urine culture}

The gold standard for UTI diagnosis is urine culture as recommended by international huidelines [8]. Urine specimens may be collected either as a clean-catch midstream sample, from an indwelling urethral catheter or a suprapubic aspiration from a suprapubic catheter [14]. Urine microscopy provides information about the cell types in the urine and the term leukocyturia is used when there are greater than 10 white blood cells per high-power field (x400) in the re-suspended sediment of a centrifuged aliquot of urine, or per $\mathrm{mm}^{3}$ in unspun urine [14]. Urine is routinely cultured on nutrient-dense medium and incubated over 2 days before it is said to be negative.

The definition for significant bacteriuria depends upon how the urine sample is collected. For a routine clean-catch midstream sample, a significant growth of bacteria is defined by counts of $>10^{5}$ colony forming units $(\mathrm{CFU}) / \mathrm{mL}$ [16]. For a urethral catheter urine sample, $>10^{4}$ CFU/mL may be considered relevant [16] whereas for a suprapubic bladder puncture specimen, any count of bacteria is relevant [16].

Escherichia coli is the most common isolated pathogen (50\%), followed by Pseudomonas aeruginosa (15\%), Acinetobacter (15\%), Enterococcus faecalis $(6 \%)$ and multiorganismic infections [17],[18]. This is in contrast to the pathogens cultured in simple UTIs which are Escherichia coli (90\%), Proteus (5\%) and Klebsiella (4\%) [16]. Organisms such as Chlamydia and Ureaplasma urealyticum should be considered in patients with leukocyturia and negative routine cultures. 
Patients using catheters may often consistently have uropathogens cultured form their urine (known as asymptomatic bacteriuria) [16] and therefore, periodically sending urine samples for culture should be discouraged in the absence of fresh neurological or urological symptoms.

Positive urine culture should be followed by identification of sensitivities to antibiotics in order to decide treatment. By definition, a multiresistant microorganism cultured is resistant to three or more different antibiotics [19].

\section{Recurrent UTIs}

Some PwMS experience recurrent UTIs and though a standard definition is lacking, according to the guidelines of the European Association of Urology, a patient reporting at least three episodes of UTIs documented by culture in the past 12 months, or two UTI episodes in the preceding six months is said to have recurrent UTIs [16]. Risk factors for recurrent UTIs have been poorly studied, however it is thought that the presence of LUT dysfunction such as detrusor overactivity, detrusor sphincter dyssynergia or incomplete bladder emptying with large post-void residual volumes, the use of urinary catheters, the presence of vesicoureteric reflux, female gender and the use of immunosuppressive treatment increases the likelihood for UTIs [2],[3],[21]. Increasing functional dependence is associated with greater difficulties in toileting and personal hygiene and may increase the risk for UTIs. Urinary retention increases the risk for UTIs [2],[8] because of the multiplication of bacteria due to stagnation of urine in the bladder. It is therefore recommended that patients with high post-void residuals should regularly perform ISC to reduce the risks for UTI [1],[8],[20].

However, the use of a catheter is in itself associated with a risk for developing UTIs [21] by compromising the host defences in several ways. Catheterisation obviates the protection from 
infections afforded by the length of urethra between the external and internal urethral openings as bacteria are deposited directly into the bladder [22]. A glycosaminoglycan layer coats the mucosal surface of the bladder in health, and a catheter traumatises the bladder wall resulting in urothelial damage and compromise to this layer [23]. This increases the chances for bacterial penetration across the bladder wall. Mechanical cleansing of the LUT by natural voiding is yet another defence against infections that is compromised by an indwelling catheter. The biofilm that forms on the surface of the indwelling catheter creates an ideal environment for the growth of microorganisms and contributes to the emergence of resistance to host defences and antimicrobials. For this reason, intermittent cathterisation is associated with a reduced risk for UTIs compared to an indwelling catheter. Adopting a sterile technique (versus clean) further reduces the risk for UTI and/or bacteriuria compared with clean intermittent catheterisation, however this is usually not a feasible option in routine practice [8].

\section{Management of UTIs in patients with MS}

\section{Asymptomatic bacteriuria}

Asymptomatic bacteriuria in PwMS should not be treated (figure 1) [16] except in the context of an acute MS relapse [24]. Treating asymptomatic bacteriuria is not associated with better outcomes, and also results in resistant bacterial strains [16].

\section{Symptomatic UTI}


Antibiotic treatment is recommended for symptomatic UTIs (figure 1). Empirical treatment is usually initiated with broad-spectrum antibiotics based on local susceptibility patterns or the patient's previous microbiology results, and the choice of antibiotics may need to be adjusted according to the results of urine culture (figure 1). There is no recommendation in the literature about the duration of treatment. By definition, UTIs occurring in PwMS are complicated, as they are associated with structural or functional abnormalities of the genitourinary tract in the presence of an underlying disease and therefore the risk for failing therapy is greater [16]. A 5-7 day course of antibiotic treatment is generally advised, which may be extended to 14 days according to the severity of infection [8]. Symptomatic complicated UTI in the setting of an indwelling catheter should be treated with a narrow spectrum antibiotic whenever possible, and modified based on culture and sensitivity results [16].

\section{Suspected UTI in the patient with an MS relapse}

In the case of suspected UTI in the context of a relapse, corticosteroid therapy should not be delayed and the initiation of antibiotic treatment is influenced by the results of urinalysis. Rakusa et al [24] presented a urinalysis algorithm for UTI prior to initiation of corticosteroid therapy in PwMS who experienced relapse. In this study, the use of the urinalysis (positive if either leukocyte esterase or nitrites positive) had a specificity of $78 \%$ and a sensitivity of $75 \%$. Negative predictive value of urinalysis was $96 \%$. Neither the $3 \%$ of patients with false negative urinalysis nor the $18 \%$ false positive patients who were unnecessarily treated with antibiotics for $48 \mathrm{~h}$ had any clinical adverse events. PwMS with systemic features of infection were excluded eg fever. Mahadeva et al reported a similar result in a large audit and also supported the initiation of corticosteroid treatment administered simultaneously with antibiotic treatment for the UTI, with the same caveats. [25]. 


\section{Recurrent UTIS}

Guidance for the management of recurrent UTIs is lacking. Urinary tract infections may exacerbate MS-related lower urinary tract (LUT) symptoms such as urinary urgency, frequency and incontinence and infections may also predispose to complications of the upper urinary tract including stones, pyelonephritis and vesicoureteric reflux [1].

\section{- Management of lower urinary tract dysfunction}

The National Institute for Health Care and Excellence (NICE) guidelines state that PwMS and recurrent UTIs should be assessed by specialists for risk factors and offered appropriate treatment and guidance [7]. The occurrence of recurrent UTIs in PwMS may indicate suboptimal management of underlying LUT dysfunction, e.g. high bladder pressure during storage and voiding and incomplete bladder emptying.

Different options are available to treat overactive bladder in MS patients, including oral antimuscarinic agents, tibial nerve stimulation, intradetrusor botulinum toxin A injections and sacral neuromodulation [1],[20]. Intradetrusor botulinum toxin A injections are highly effective for the management of detrusor overactivity in MS [26] and in fact, treatment has been shown to reduce the incidence of symptomatic UTIs. The reasons for this are uncertain, however the treatment is associated with an improvement in urodynamic parameters with an improved reservoir capacity at low pressure [27]. Ultrasonography of the bladder is regularly performed to search for contributing factors, such as bladder stones. The need for an indwelling catheter should be reviewed [8] and if required, the type of catheter used may be relevant as a hydrophilic catheter is associated with a lower rate of UTIs, at least in men [28]. Long-term indwelling urethral catheter should be avoided because of a higher risk of UTIs, urethral complications, bladder stones and cancer, and rather the option of ISC or suprapubic catheter should be considered [16]. Strategies to minimise infection should be rigorously 
followed including catheter insertion under antiseptic conditions, use of a closed catheter drainage systems with dependent drainage and regular changes of long-term catheters before likely blockage. Patients with a large bladder capacity low pressure bladder may be managed using a flip-flow valve attached to the catheter after assessing suitability, thus avoiding the closed drainage bag. Often these measures are insufficient to prevent UTIs and more specific preventative strategies may be required. However, these strategies remain largely unvalidated with a lack of high grade recommendation in international guidelines.

\section{- Antibiotic prophylaxis}

Continuous antibiotic prophylaxis for 6-12 months has been shown to be effective in reducing the rate of UTI during prophylaxis when compared to placebo in women without neurological disease [29]. However their use in neurological patients with LUT dysfunction is uncertain. The use of oral antimicrobial prophylaxis for preventing UTIs in this group was not supported in a metaanalysis by Morton et al [30] and is currently not recommended by NICE [7]. The concern about using antimicrobial prophylaxis is the emerging global problem of antimicrobial resistance as a two-fold increase in resistant bacteria was reported with this approach [30]. The results of a recent observational prospective pilot study by Salomon et al [31] however suggests that a weekly oral cyclic antibiotic (WOCA) regimen to prevent UTIs is both safe and efficacious in adult spinal-cord injured patients with LUT dysfunction performing ISC. The WOCA regimen consists of alternate administration of two antibiotics once a week over a period of at least 2 years. The antibiotics chosen were effective for preventing UTIs, with a dramatic reduction in the incidence of UTIs from 9.4 symptomatic infections per patient-year and 197 episodes of febrile UTI responsible requiring 45 hospitalizations before intervention, to 1.8 symptomatic UTIs per patient-year, 19 episodes of febrile UTI and 7 hospitalizations afterwards. No severe adverse events and no new cases of 
colonization with multidrug resistant bacteria were reported [31]. This regimen requires to be studied in PwMS.

\section{- Cranberry}

The use of cranberry for UTI prophylaxis in MS is an attractive option, as it appears to be a safe and natural choice. However, there are a few studies that have evaluated cranberry in preventing UTIs and the results have been mixed. A meta-analysis of randomized controlled trials including heterogenous populations (patients with neurogenic bladder, elderly, pregnant women, women with recurrent UTIs and children) found that cranberry could reduce the number of UTIs by two-fold [32] whereas a Cochrane review of 24 studies, with a total of 4,473 subjects, revealed that cranberry products were of no benefit compared to placebo in most populations [33], including spinal cord injured patients (14.3\%).

A recent multicentre, randomized, placebo-controlled, double-blind trial in MS using cranberry extract could not demonstrate a reduction in UTI occurrence in 171 patients [33]. Therefore, the use of cranberry extracts for preventing UTIs is not supported by high-level evidence, though in practice is widely used by PwMS.

\section{- D-Mannose}

D- Mannose is a natural food supplement and is thought to work in a similar fashion as cranberry. Indeed, D-mannose binds to FimH at the tip of type 1 fimbria of enteric bacteria. During bacterial colonization, FimH binds to carbohydrate-containing glycoprotein receptors on the epithelium of the urinary tract. D-mannose shares similarity in structure to these urothelial glycoprotein receptors and therefore acts by competitively inhibiting bacterial adherence to carbohydrate-containing glycoprotein receptors on the epithelium of the urinary tract. 
In a randomized-controlled trial, [35] the daily intake of D-mannose powder $2 \mathrm{~g}$ for recurrent UTI prophylaxis in women without neurological disease was evaluated and a $45 \%$ relative risk reduction was reported, with results similar to the use of preventative nitrofurantoin. The use of D-Mannose in PwMS is currently being studied in an on-going study (ClinicalTrials.gov NCT02490046).

\section{- Other approaches}

A variety of approaches have been evaluated but without proven efficacy. Bladder irrigation and methenamine hippurate has been studied, however there is insufficient evidence to support its use [36],[37]. The inoculation of apathogenic E. coli strains into the bladder has shown promise in initial randomized controlled studies, however further studies are required before recommendations could be made [38].

\section{Conclusion}

Urinary tract infections are a major concern for PwMS and pose a challenge to the treating physician. Despite the significant impact that UTIs have, there is a dearth of studies around the diagnosis and management of UTIs. This review provides an overview of the diagnosis, treatment and prevention of UTIs in people with MS. Known facts are the usefulness of urinalysis using to exclude a UTI and the non-treatment of asymptomatic UTIs except in the context of an acute MS relapse. Several options exist for the treatment of recurrent UTIs in MS, however none has been validated. Further studies targeting this specific population suffering from UTIs are needed.

\section{Abbreviations}


UTI: urinary tract infection

MS: multiple sclerosis

LUT: lower urinary tract

ISC: intermittent self-catheterisation

\section{Figure}

Figure 1. Flowchart for the testing for UTIs in patients with multiple sclerosis

\section{Acknowledgement:}

The work was undertaken at UCLH/UCL Institute of Neurology and is supported in part by funding from the United Kingdom's Department of Health NIHR Biomedical Research Centres funding scheme.

The authors thank Gwen Gonzales for her useful comments.

\section{Funding:}

VP was supported by the European Urological Scholarship Programme.

\section{Conflict of interest:}

VP: none to declare

MP: none to declare

CC: none to declare

BP: none to declare

$\mathrm{CH}$ : none to declare

JC: none to declare

JNP: none to declare 


\section{References}

1. de Sèze M, Ruffion A, Denys $\mathbf{P}$, et al. The neurogenic bladder in multiple sclerosis: review of the literature and proposal of management guidelines. Mult. Scler. J. 2007; 13(7):915-928.

2. Gallien P, Robineau S, Nicolas B, et al. Vesicourethral dysfunction and urodynamic findings in multiple sclerosis: a study of 149 cases. Arch. Phys. Med. Rehabil. 1998; 79(3):255-257.

3. Jick SS, Li L, Falcone GJ, Vassilev ZP, Wallander M-A. Epidemiology of multiple sclerosis: results from a large observational study in the UK. J. Neurol. 2015; 262(9):20332041.

4. Marrie RA, Elliott L, Marriott J, et al. Dramatically changing rates and reasons for hospitalization in multiple sclerosis. Neurology. 2014; 83(10):929-937.

5. Manack A, Motsko SP, Haag-Molkenteller C, et al. Epidemiology and healthcare utilization of neurogenic bladder patients in a US claims database. Neurourol. Urodyn. 2011; 30(3):395-401.

6. Buljevac D, Flach HZ, Hop WCJ, et al. Prospective study on the relationship between infections and multiple sclerosis exacerbations. Brain. 2002; 125(5):952-960.

7. Anon. National Institute for Health \& Care Excellence (NICE). Urinary incontinence in neurological disease: management of lower urinary tract dysfunction in neurological disease. [CG148]. London: NICE; 2012.

8. Anon. EAU guidelines on neuro-urology. Uroweb. Available at: http://uroweb.org/guideline/neuro-urology/ [Accessed September 2, 2015].

9. Edlich RF, Westwater JJ, Lombardi SA, Watson LR, Howards SS. Multiple sclerosis and asymptomatic urinary tract infection. J. Emerg. Med. 1990; 8(1):25-28.

10. Goetz LL, Klausner AP. Strategies for Prevention of Urinary Tract Infections in Neurogenic Bladder Dysfunction. Phys. Med. Rehabil. Clin. N. Am. 2014; 25(3):605-618. 11. Massa LM, Hoffman JM, Cardenas DD. Validity, Accuracy, and Predictive Value of Urinary Tract Infection Signs and Symptoms in Individuals With Spinal Cord Injury on Intermittent Catheterization. J. Spinal Cord Med. 2009; 32(5):568-573.

12. Devillé WL, Yzermans JC, van Duijn NP, et al. The urine dipstick test useful to rule out infections. A meta-analysis of the accuracy. BMC Urol. 2004; 4:4.

13. Tuel SM, Meythaler JM, Cross LL, McLaughlin S. Cost-effective screening by nursing staff for urinary tract infection in the spinal cord injured patient. Am. J. Phys. Med. Rehabil. Assoc. Acad. Physiatr. 1990; 69(3):128-131.

14. Anon. EAU guidelines on urological Infections. Uroweb. Available at:

http://uroweb.org/guideline/urological-infections/ [Accessed October 8, 2015].

15. Anon. The prevention and management of urinary tract infections among people with spinal cord injuries. National Institute on Disability and Rehabilitation Research Consensus Statement. January 27-29, 1992. J. Am. Paraplegia Soc. 1992; 15(3):194-204.

16. Anon. Guidelines on urological infections. European Association of Urology. 2015.

17. Shigemura K, Takase R, Osawa K, et al. Emergence and prevention measures for multidrug resistant Pseudomonas aeruginosa in catheter-associated urinary tract infection in spinal cord injury patients. Spinal Cord. 2015; 53(1):70-74.

18. Jahromi MS, Mure A, Gomez CS. UTIs in patients with neurogenic bladder. Curr. Urol. Rep. 2014; 15(9):433.

19. Magiorakos A-P, Srinivasan A, Carey RB, et al. Multidrug-resistant, extensively drugresistant and pandrug-resistant bacteria: an international expert proposal for interim standard definitions for acquired resistance. Clin. Microbiol. Infect. Off. Publ. Eur. Soc. Clin.

Microbiol. Infect. Dis. 2012; 18(3):268-281.

20. Fowler CJ, Panicker JN, Drake M, et al. A UK consensus on the management of the 
bladder in multiple sclerosis. J. Neurol. Neurosurg. Psychiatry. 2009; 80(5):470-477.

21. Edokpolo LU, Stavris KB, Foster HE. Intermittent Catheterization and Recurrent Urinary Tract Infection in Spinal Cord Injury. Top. Spinal Cord Inj. Rehabil. 2012; 18(2):187-192.

22. Neal DE. Host defense mechanisms in urinary tract infections. Urol. Clin. North Am. 1999; 26(4):677-686, vii.

23. Delnay KM, Stonehill WH, Goldman H, Jukkola AF, Dmochowski RR. Bladder histological changes associated with chronic indwelling urinary catheter. J. Urol. 1999; 161(4):1106-1108; discussion 1108-1109.

24. Rakusa M, Murphy O, McIntyre L, et al. Testing for urinary tract colonization before high-dose corticosteroid treatment in acute multiple sclerosis relapses: prospective algorithm validation. Eur. J. Neurol. 2013; 20(3):448-452.

25. Mahadeva A, Tanasescu R, Gran B. Urinary tract infections in multiple sclerosis: under-diagnosed and under-treated? A clinical audit at a large University Hospital. Am. J. Clin. Exp. Immunol. 2014; 3(1):57-67.

26. Cruz F, Nitti V. Chapter 5: Clinical data in neurogenic detrusor overactivity (NDO) and overactive bladder (OAB). Neurourol. Urodyn. 2014; 33(S3):S26-S31.

27. Gamé X, Castel-Lacanal E, Bentaleb Y, et al. Botulinum Toxin A Detrusor Injections in Patients with Neurogenic Detrusor Overactivity Significantly Decrease the Incidence of Symptomatic Urinary Tract Infections. Eur. Urol. 2008; 53(3):613-619.

28. Cardenas DD, Hoffman JM. Hydrophilic catheters versus noncoated catheters for reducing the incidence of urinary tract infections: a randomized controlled trial. Arch. Phys. Med. Rehabil. 2009; 90(10):1668-1671.

29. Albert X, Huertas I, Pereiró II, et al. Antibiotics for preventing recurrent urinary tract infection in non-pregnant women. Cochrane Database Syst. Rev. 2004; (3):CD001209.

30. Morton SC, Shekelle PG, Adams JL, et al. Antimicrobial prophylaxis for urinary tract infection in persons with spinal cord dysfunction. Arch. Phys. Med. Rehabil. 2002;

83(1):129-138.

31. Salomon J, Denys P, Merle C, et al. Prevention of urinary tract infection in spinal cordinjured patients: safety and efficacy of a weekly oral cyclic antibiotic (WOCA) programme with a 2 year follow-up--an observational prospective study. J. Antimicrob. Chemother. 2006; 57(4):784-788.

32. Wang C-H, Fang C-C, Chen N-C, et al. Cranberry-containing products for prevention of urinary tract infections in susceptible populations: a systematic review and meta-analysis of randomized controlled trials. Arch. Intern. Med. 2012; 172(13):988-996.

33. Jepson R, Craig J, Williams G. CRanberry products and prevention of urinary tract infections. JAMA. 2013; 310(13):1395-1396.

34. Gallien P, Amarenco G, Benoit N, et al. Cranberry versus placebo in the prevention of urinary infections in multiple sclerosis: a multicenter, randomized, placebo-controlled, double-blind trial. Mult. Scler. J. 2014; 20(9):1252-1259.

35. Kranjčec B, Papeš D, Altarac S. d-mannose powder for prophylaxis of recurrent urinary tract infections in women: a randomized clinical trial. World J. Urol. 2013; 32(1):79-84.

36. Waites KB, Canupp KC, Roper JF, Camp SM, Chen Y. Evaluation of 3 Methods of Bladder Irrigation to Treat Bacteriuria in Persons With Neurogenic Bladder. J. Spinal Cord Med. 2006; 29(3):217-226.

37. Lee BSB, Bhuta T, Simpson JM, Craig JC. Methenamine hippurate for preventing urinary tract infections. Cochrane Database Syst. Rev. 2012; 10:CD003265.

38. Darouiche RO, Green BG, Donovan WH, et al. Multicenter randomized controlled trial of bacterial interference for prevention of urinary tract infection in patients with neurogenic bladder. Urology. 2011; 78(2):341-346. 